\title{
Vertex Splitting In Dags And Applications To Partial Scan Designs And Lossy Circuits
}

\author{
Doowon Paik+ \\ Sudhakar Reddy++ \\ Sartaj Sahni+ \\ University of Florida \\ University of Iowa \\ University of Florida
}

\begin{abstract}
Directed acyclic graphs (dags) are often used to model circuits. Path lengths in such dags represent circuit delays. In the vertex splitting problem, the objective is to determine a minimum number of vertices to split so that the resulting dag has no path of length $\delta$. This problem has application to the placement of flip-flops in partial scan designs, placement of latches in pipelined circuits, placement of signal boosters in lossy circuits and networks, etc. Several simplified versions of this problem are shown to be $N P$-hard. A linear time algorithm is obtained for the case when the dag is a tree. A backtracking algorithm and heuristics are developed for general dags and experimental results using dags obtained from ISCAS benchmark circuits are obtained.
\end{abstract}

\section{KEYWORDS and PHRASES}

Partial-scan designs, flip-flop selection, sequential circuits, lossy circuits and networks, pipelined circuits, NP-hard

\footnotetext{
+ Research supported, in part, by the National Science Foundation under grants DCR-84-20935 and MIPS-86-17374. ++ Research supported, in part, by the SDIO/IST Contract No. N00014-90-J-1793 managed by US Office of Naval Research.
} 


\section{Introduction}

In order to achieve high fault coverage in sequential circuits they are often designed to be easily testable. The current method of choice is the scan-design. In test mode all flip-flops in a sequential circuit, using scan-design, are connected into one or more shift registers. This allows one to set the contents of the flip-flops to the desired state as well as to observe the states of the flipflops. As the complexity of logic circuits grows, the overhead for full scan-designs may become unacceptable. For such situations, partial-scan designs have been proposed. In partial-scan designs only a selected subset of the flip-flops in a sequential circuit are included in the scanpath. Several methods to choose the flip-flops to be included in the scan-path have been proposed [CHEN90], [GUPT90], [LEE90]. One of these proposals gives a method to use the structural information in a sequential circuit to determine the flip-flops to be placed in a scan-path [CHEN90]. We briefly discuss this method.

A sequential circuit is represented by a directed graph (digraph) called S-graph. Each flipflop in a sequential circuit is represented by a node in the S-graph. A directed edge exists in the S-graph from node $i$ to node $j$ if the state of the flip-flop represented by node $j$ depends on the state of the flip-flop represented by node $i$ (that is ,there is a path, through combinational logic, in the circuit from the output of flip-flip $i$ to the input of flip-flop $j$ ). Figure 1 is an example of a Sgraph. Empirical evidence suggests that the existence of cycles and the maximum path length between nodes of the S-graph increase the complexity of deriving tests for sequential circuits. It was therefore suggested in [CHEN90] to include a minimum subset of flip-flops into a scan-path such that the resulting $\mathrm{S}$-graph is cycle-free and the maximum distance between a pair of nodes is small.

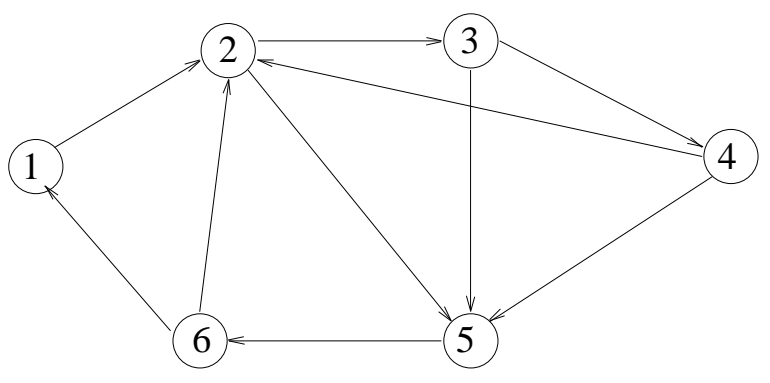

Figure 1: An example S-graph.

There are several cycles in the S-graph of Figure 1. If the flip-flop corresponding to node 2 is 
included in the scan-path then one replaces node 2 with a sink node $2^{i}$ and a source node $2^{o}$ as shown in Figure 2. This transformation corresponds to the fact that the contents of flip-flops in a scan path can be set and observed in test mode. Notice that the S-graph of Figure 2 is cycle free.

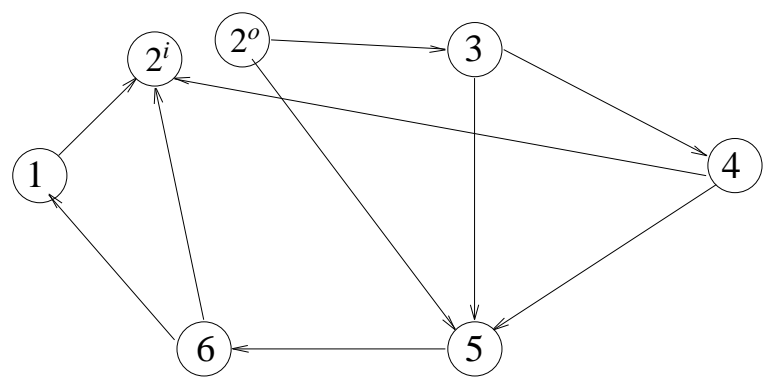

Figure 2: An acyclic S-graph for Figure 1.

The maximum distance between node $2^{o}$ and $2^{i}$ is six. If a flip-flop corresponding to node 5 is also included in the scan-path then the S-graph of Figure 3 is obtained. In this the maximum distance between any pair of nodes is less than or equal to 3 .

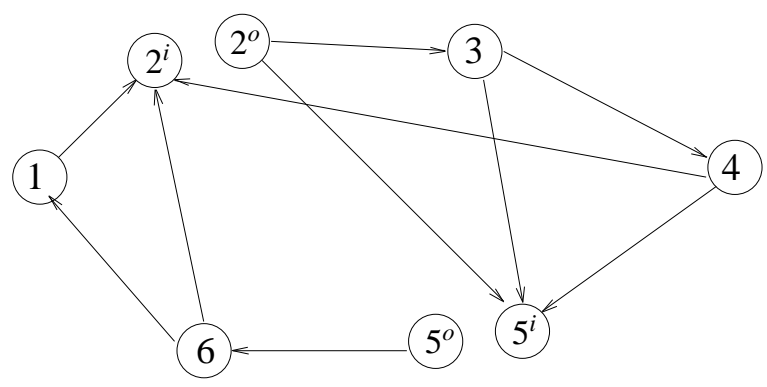

Figure 3: An S-graph with maximum distance 3.

Two step methods to select the flip-flops to be scanned were proposed in [CHEN90], [GUPT90], and [LEE90]. In the first step a minimal subset of flip-flops is selected to be included in the scan-path such that the resulting S-graph is acyclic. In the second step additional flip-flops are selected to be included in the scan path such that in the resulting S-graph the maximum 
distance between any pair of nodes is less than or equal to a specified number $\delta$. This second step can be modeled as a vertx splitting problem on directed acyclic graphs (dags).

In this paper we study solutions to the problem of finding a minimum number of nodes, in a dag, to be split such that the maximum distance between any two nodes in the resulting digraph is less than or equal to a pre-specified value $\delta$. The dags we consider are more general than the ones that arise from S-graphs. We permit each edge in the dag to have a positive integral weight instead of requiring all edges to have unit weight. This generalization can be shown to have application in the placement of latches in pipelined circuits and in the placement of signal boosters in lossy circuits.

In Section 2, we introduce the terminology we shall use in the remainder of this paper. The NP-hard results are developed in Section 3 and the linear time algorithm for tree dags is given in Section 4. A backtracking algorithm and heuristics for the dag vertex splitting problem are proposed in Section 5 and 6, respectively. Section 7 reports on experiments with the ISCAS benchmark circuits. It should be noted that a linear time algorithm for series-parallel dags is easily derived from the linear time dag vertex deletion algorithm of [PAIK90].

\section{Terminology}

Let $G=(V, E, w)$ be a weighted directed acyclic graph (wdag) with vertex set $V$, edge set $E$, and edge weighting funtion $w . w(i, j)$ is the weight of the edge $\langle i, j\rangle \in E . w(i, j)$ is a positive integer for $\langle i, j\rangle \in E$ and $w(i, j)$ is undefined if $\langle i, j\rangle \notin E$. A source vetex is a vertex with zero indegree while a sink vetex is a vertex with zero out-degree. The delay, $d(P)$, of the path $P$ is the sum of the weights of the edges on that path. The delay, $d(G)$, of the graph $G$ is the maximum path delay in the graph, i.e.,

$$
d(G)=\max _{P \text { in } G}\{d(P)\}
$$

Let $G / X$ be the wdag that results when each vertex $v$ in $X$ is split into two $v^{i}$ and $v^{o}$ such that all edges $\langle v, j\rangle \in E$ are replaced by edges of the form $\left\langle v^{o}, j\right\rangle$ and all edges $\langle i, v\rangle \in E$ are replaced by edges of the form $\left\langle i, v^{i}\right\rangle$. I.e., outbound edges of $v$ now leave vertex $v^{o}$ while the inbound edges of $v$ now enter vertex $v^{i}$. Figure 3 shows the result, $G / X$, of splitting the vertex 5 of the dag of Figure 2. The dag vertex splitting problem (DVSP) is to find a least cardinality vertex set $X$ such that $d(G / X) \leq \delta$, where $\delta$ is a prespecified delay. For the dag of Figure 2 and $\delta=3, X=$ $\{5\}$ is a solution to the DVSP problem.

Lemma 1: Let $G=(V, E, w)$ be a weighted dag and let $\delta$ be a prespecified delay value. Let MaxEdgeDelay $=\max _{\langle i, j\rangle \in E}\{w(i, j)\}$. Then the DVSP has a solution iff $\delta \geq$ MaxEdgeDelay. 
Proof: Vertex splitting does not eliminate any edges. So, there is no $X$ such that $d(G / X)<$ MaxEdgeDelay. Further, $d(G / V)=$ MaxEdgeDelay. So, for every $\delta \geq$ MaxEdgeDelay, there is a least cardinality set $X$ such that $d(G / X) \leq \delta$.

\section{Complexity Results}

If $w(i, j)=1$ for every edge in the wdag, then the edge weighting function $w$ is said to be a unit weighting function and we say that $G$ has unit weights. In this section we show that the following problems are $N P$-hard.

1. DVSP for unit weight graphs with $\delta \geq 2$.

2. DVSP for unit weight multistage graphs with $\delta \geq 4$. (in a multistage graph the vertices are divided into an ordered set of stages and each edge goes from a vertex in one stage to one in the next stage).

Since unit weight wdags are just a special case of general wdags, the results obtained imply the $N P$-hardness of the corresponding problems with the unit weight constraint removed.

\subsection{Unit Weight DVSP}

We shall show that the known NP-complete problem 3SAT can be solved in polynomial time if the unit weight DVSP with $\delta \geq 2$ can.

\section{SAT Problem[GARE79]}

Input: A boolean function $F=C_{1} C_{2} \cdots C_{n}$ in $n$ variables $x_{1}, x_{2}, \ldots, x_{n}$. Each clause $C_{i}$ is the disjunction of exactly three literals.

Output: "Yes" if there is a binary assignment for the $n$ variables such that $F=1$. "No" otherwise.

For each instance $F$ of 3SAT, we construct an instance $G_{F}$ of the unit weight DVSP such that from the size of the solution to $G_{F}$ we can determine, in polynomial time, the answer to the 3SAT problem for $F$. This construction employs two unit weight dag subassemblies: variable subassembly and clause subassembly.

\section{Variable Subassembly}

Figure 4(a) shows a chain with $\delta-1$ vertices. This chain is represented by the schematic of Figure 4(b). The variable subassembly, VS(i), for variable $x_{i}$ is given in Figure 4(c). This is obtained by combining together three copies of the chain $H_{\delta-1}$ with another chain that has three vertices. Thus, the total number of vertices in the variable subassembly $V S(i)$ is $3 \delta$. Note that $d(V S(i))=\delta+1$. Also, note that if $d(V S(i) / X) \leq \delta$, then $|X| \geq 1$. The only $X$ for which $|X|=1$ and 


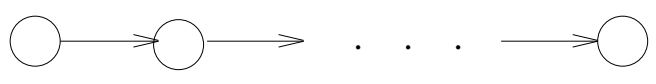

(a) Chain with $\delta$ - 1 vertices

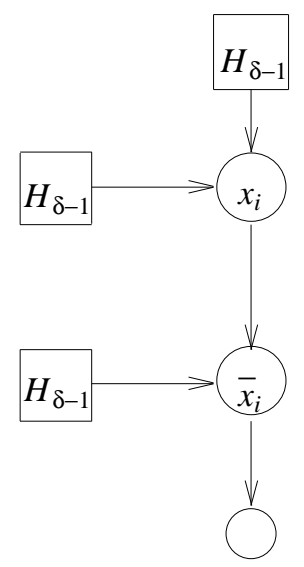

(c) $\quad V S(i)$
$H_{\delta-1}$

(b) Schematic

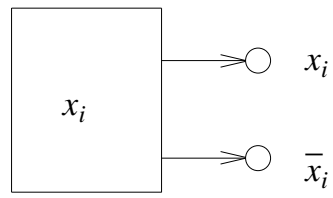

(d) Schematic

Figure 4: Variable subassembly for DVSP.

$d(\operatorname{VS}(i) / X) \leq \delta$ are $X=\left\{x_{i}\right\}$ and $X=\left\{\bar{x}_{i}\right\}$. Figure 4(d) shows the schematic for $V S(i)$.

\section{Clause Subassembly}

The clause subassembly $C S(j)$ is obtained by connecting together four $\delta-1$ vertex chains with another three vertex subgraph as shown in Figure 5(a). The schematic for $C S(j)$ is given in Figure 5(b). The number of vertices in $C S(j)$ is $4 \delta-1$ and $d(C S(j))=2 \delta$. One may easily verify that if $|X|=1$, then $d(C S(j) / X)>\delta$. So, if $d(C S(j) / X) \leq \delta$, then $|X|>1$. Since $\delta \geq 2$, the only $X$ with $|X|=2$ for which $d(C S(j) / X) \leq \delta$ are such that $X \subseteq\left\{l_{j 1}, l_{j 2}, l_{j 3}\right\}$. Furthermore, every $X \subseteq$ $\left\{l_{j 1}, l_{j 2}, l_{j 3}\right\}$ with $|X|=2$ results in $d(C S(j) / X) \leq \delta$.

To construct $G_{F}$ from $F$, we use $n V S(i)$ 's, one for each variable $x_{i}$ in $F$ and $m C S(j)$ 's, one for each clause $C_{j}$ in $F$. There is a directed edge from vertex $x_{i}\left(\bar{x}_{i}\right)$ of $V S(i)$ to vertex $l_{j k}$ of $C S(j)$ iff $x_{i}$ $\left(\bar{x}_{i}\right)$ is the $k^{\prime}$ th literal of $C_{j}$ (we assume the three literals in $C_{j}$ are ordered). For the case $F=$ $\left(x_{1}+\bar{x}_{2}+\bar{x}_{4}\right)\left(\bar{x}_{1}+\bar{x}_{3}+\bar{x}_{4}\right)\left(x_{1}+x_{2}+x_{3}\right)$, the $G_{F}$ of Figure 6 is obtained.

Since the total number of vertices in $G_{F}$ is $3 \delta n+(4 \delta-1) m$, the construction of $G_{F}$ can be done in polynomial time for any fixed $\delta$. 

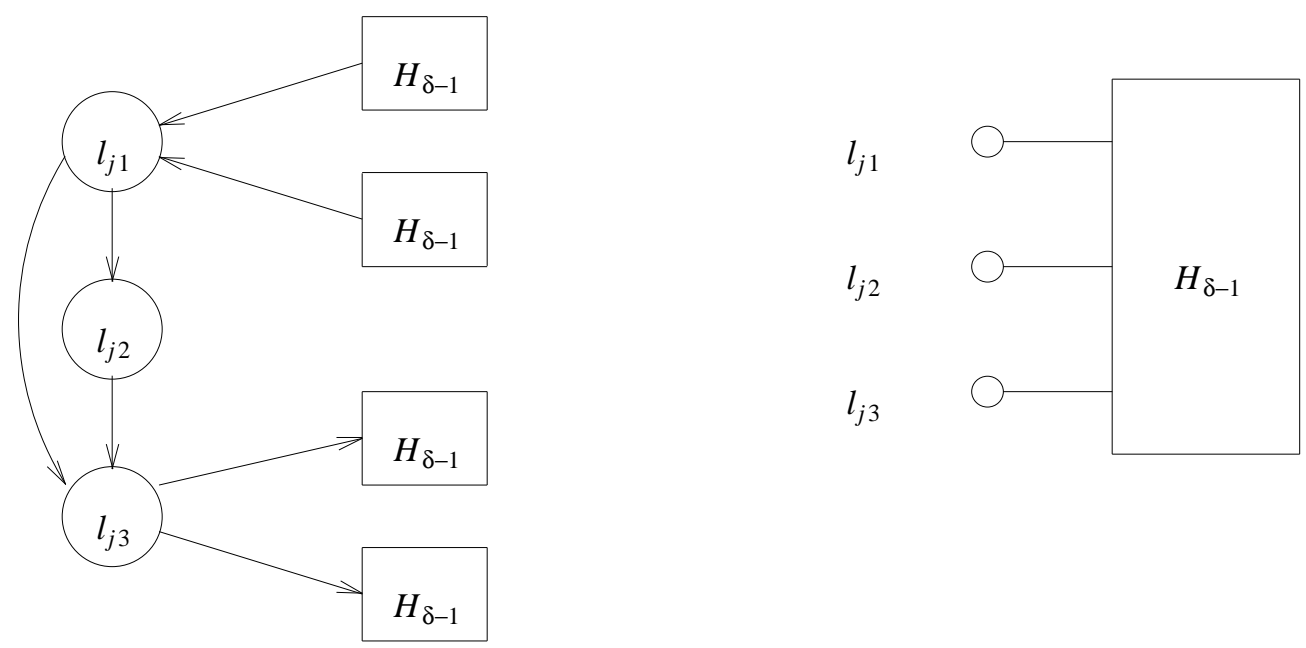

(a) $\quad C S(j)$

(b) Schematic

Figure 5: Clause assembly for DVSP.

Theorem 1: Let $F$ be an instance of 3SAT and let $G_{F}$ be the instance of unit weight DVSP obtained using the above construction. For $\delta \geq 2, F$ is satisfiable iff there is a vertex set $X$ such that $d\left(G_{F} / X\right) \leq \delta$ and $|X|=n+2 m$.

Proof: If $F$ is satisfiable then there is a binary assignment to the $x_{i}$ 's such that $F$ has value 1 . Let $b_{1}, b_{2}, \ldots b_{n}$ be this ssignment. Construct a vertex set $X$ in the following way:

1. $x_{i}$ is in $X$ if $b_{i}=1$. If $b_{i}=0$, then $\bar{x}_{i}$ is in $X$.

2. >From each $C S(j)$ add exactly two of the vertices $l_{j 1}, l_{j 2}, l_{j 3}$ to $X$. These are chosen such that the literal corresponding to the vertex not chosen has value 1. Each clause has at least one literal with value 1 .

We readily see that $|X|=n+2 m$ and that $d\left(G_{F} / X\right) \leq \delta$.

Next, suppose that there is an $X$ such that $|X|=n+2 m$ and $d\left(G_{F} / X\right) \leq \delta$. >From the construction of the variable and clause assemblies and from the fact that $|X|=n+2 m$, it follows that $X$ must contain exactly one vertex from each of the sets $\left\{x_{i}, \bar{x}_{i}\right\}, 1 \leq i \leq n$ and exactly 2 from each of the sets $\left\{l_{j 1}, l_{j 2}, l_{j 3}\right\}, 1 \leq j \leq m$. Hence there is no $i$ such that both $x_{i} \in X$ and $\bar{x}_{i} \in X$ and there is no $j$ for which $l_{j 1} \in X$ and $l_{j 2} \in X$ and $l_{j 3} \in X$. Consider the Boolean assignment $b_{i}=1$ iff $x_{i} \in X$. 


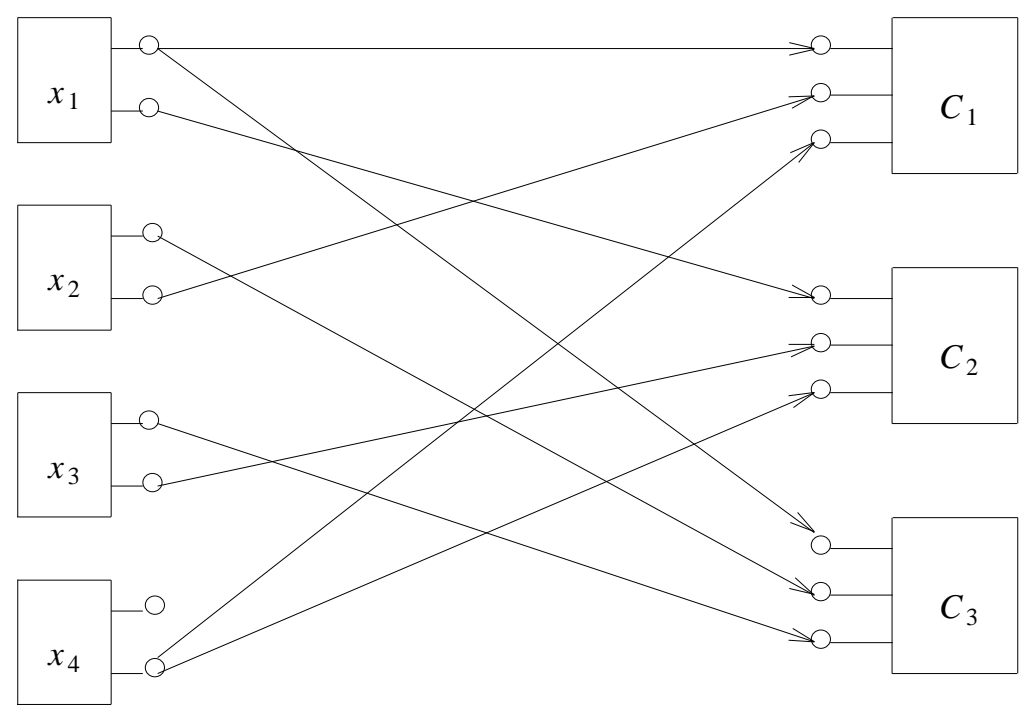

Figure 6: $G_{F}$ for $F=\left(x_{1}+\bar{x}_{2}+\bar{x}_{4}\right)\left(\bar{x}_{1}+\bar{x}_{3}+\bar{x}_{4}\right)\left(x_{1}+x_{2}+x_{3}\right)$.

Suppose that $l_{j k} \notin X$ and $l_{j k}=x_{i}\left(\bar{x}_{i}\right)$. Since $d\left(G_{F} / X\right) \leq \delta$, vertex $x_{i}\left(\bar{x}_{i}\right)$ must be split as otherwise there is a source to sink path with delay greater than $\delta$. So, $x_{i}\left(\bar{x}_{i}\right) \in X$ and $b_{i}=1(0)$. As a result, the k'th literal of clause $C_{j}$ is true. Hence, $b_{1}, \ldots b_{n}$ results in each clause having at least one true literal and $F$ has value 1 .

When $\delta=1$, the unit weight DVSP is easily solved as now every vertex that is not a source or sink has to be split.

\subsection{DVSP For Unit Weight Multistage Graphs}

A multistage graph is a dag in which the vertices are partitioned into stages and each edge connects two vertices in adjacent stages. An example is given in Figure 7. In the construction of Section 3.1, VS $(i)$ is a multistage graph but $C S(j)$ is not as the edges $\left\langle l_{j 1}, l_{j 2}\right\rangle,\left\langle l_{j 2}, l_{j 3}\right\rangle$ require $l_{j 1}$ and $l_{j 3}$ to be two stages apart while the edge $\left\langle l_{j 1}, l_{j 3}>\right.$ requires them to be one stage apart.

To show that DVSP for multistage graphs is $N P$-hard, we use the problem 2-3SAT defined as:

Input: A boolean function $F=C_{1} C_{2} \cdots C_{n}$ in $n$ variables $x_{1}, x_{2}, \ldots, x_{n}$. Each clause $C_{i}$ is the disjunction of either two or three literals. If $\left|C_{i}\right|=2$, then both literals in $C_{i}$ are either negated or unnegated. If $\left|C_{i}\right|=3$, then at least one literal of $C_{i}$ is unnegated and at least one is negated. 


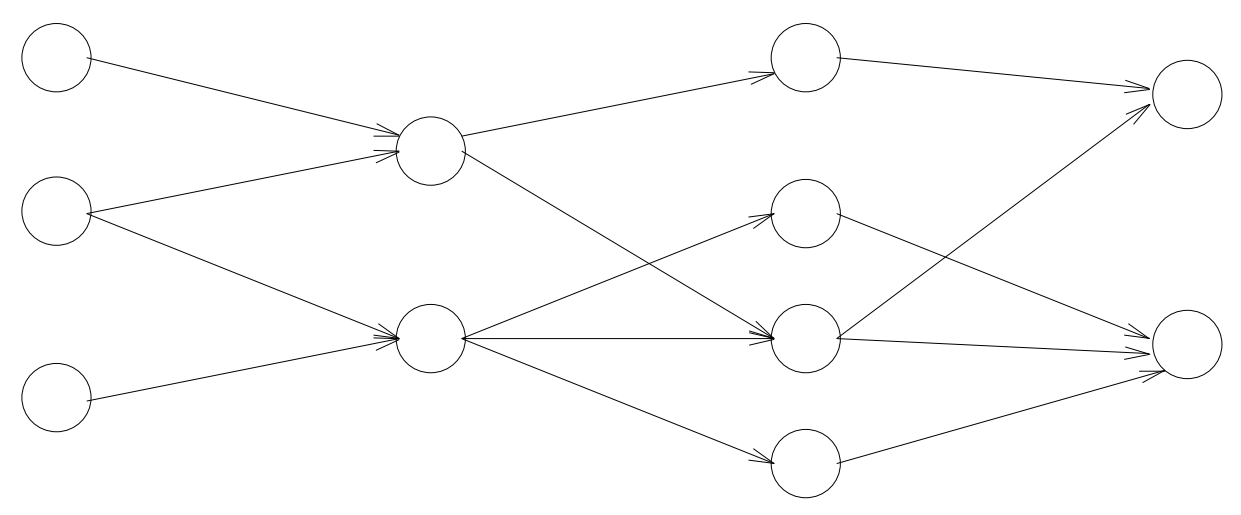

Figure 7: Example multistage graph.

Output: "Yes" iff there is a truth assignment for the $n$ variables such that $F=1$. "No" otherwise.

Theorem 2: 2-3SAT is NP-hard.

Proof: From any instance $F$ of 3SAT we can obtain, in polynomial time, an instance $H$ of 2-3SAT such that $H$ is satisfiable (i.e., has answer "yes") iff $F$ is. Consider each clause of $F$. If $C_{i}$ has only unnegated literals (say $\left.C_{i}=\left(x_{i_{1}}+x_{i_{2}}+x_{i_{3}}\right)\right)$ then replace $C_{i}$ with $\left(x_{i_{1}}+y_{1}+\bar{y}_{2}\right)\left(x_{i_{2}}+\bar{y}_{1}+y_{2}\right)\left(x_{i_{3}}\right.$ $\left.+\bar{y}_{1}+\bar{y}_{2}\right)\left(y_{1}+y_{2}\right)$, where $y_{1}$ and $y_{2}$ are new variables. If $C_{i}$ has only negated literals ( say $C_{i}=$ $\left.\left(\bar{x}_{i_{1}}+\bar{x}_{i_{2}}+\bar{x}_{i_{3}}\right)\right)$ then replace $C_{i}$ with $\left(\bar{x}_{i_{1}}+y_{1}+\bar{y}_{2}\right)\left(\bar{x}_{i_{2}}+\bar{y}_{1}+y_{2}\right)\left(\bar{x}_{i_{3}}+y_{1}+y_{2}\right)\left(\bar{y}_{1}+\bar{y}_{2}\right)$.

In this way $F$ is transformed into an instance $H$ of 2-3SAT. One may verify that $H$ is satisfiable iff $F$ is.

$>$ From an istance $F$ of 2-3SAT we can construct an istance $G_{F}$ of the multistage DVSP using the variable and clause subassemblies of Figure 8.

One may verify that for $\delta \geq 4$ :

(1) If $|X|=1$ and $d(V S(i) / X) \leq \delta$ then $X \subset\left\{x_{i}, \bar{x}_{i}\right\}$.

(2) If $|X|=2$ and $d(C S 3(j) / X) \leq \delta$ then $X \subset\left\{l_{j 1}, l_{j 2}, l_{j 3}\right\}$.

(3) If $|X|=1$ and $d(C S 2(j) / X) \leq \delta$ then $X \subset\left\{l_{j 1}, l_{j 2}\right\}$.

The construction of $G_{F}$ is similar to that used in Section 3.1 except that the variable and clause subassemblies of Figure 8 are used. In case $\left|C_{j}\right|=2$, a modified $\operatorname{CS} 2(j)$, subassembly as in Figure 9(a) is used. If $\left|C_{j}\right|=3$, then a modified $C S 3(j)$ is used. This modification is now 


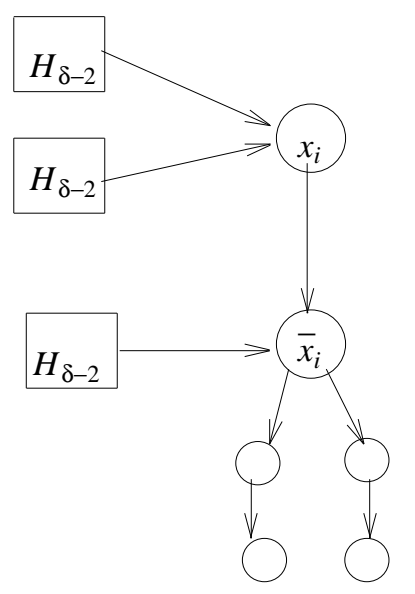

(a) $V S(i)$

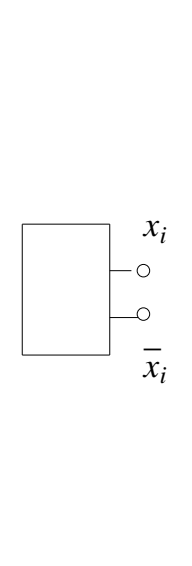

(b) Schematic

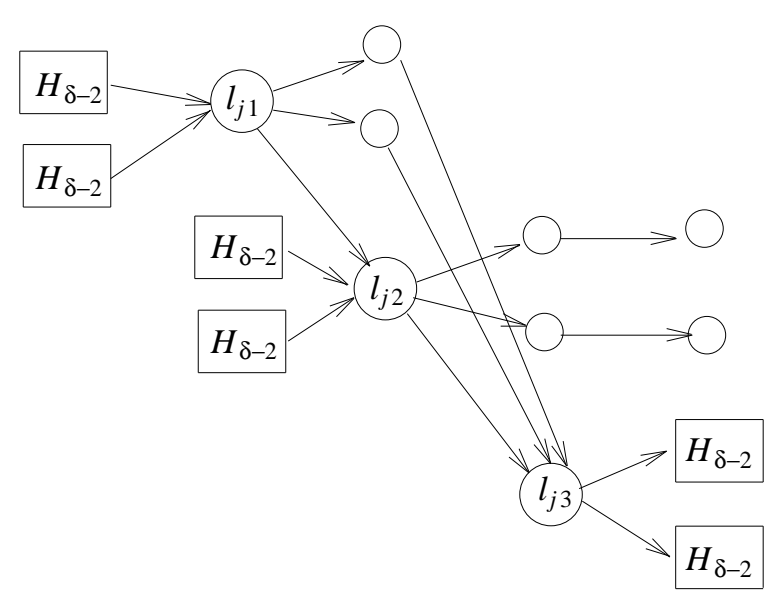

(c) $\operatorname{CS} 3(j)$ for $\left|C_{j}\right|=3$

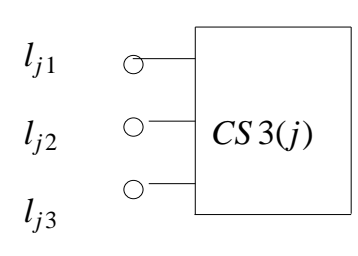

(d) Schematic

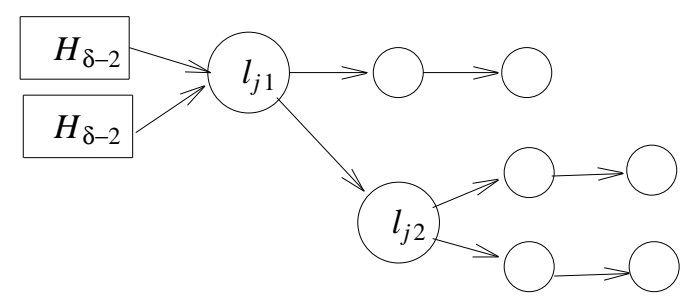

(e) $\operatorname{CS} 2(j)$ for $\left|C_{j}\right|=2$

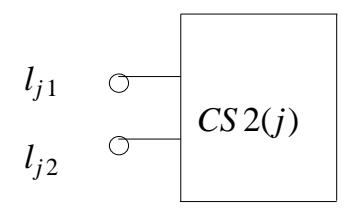

(f) Schematic

Figure 8: Subassemblies for DVSP multistage graph.

described. Suppose the literals in $C_{j}$ are ordered so that the unnegated ones come first. If $C_{j}$ has two unnegated literals, use the clause subassembly of Figure 9(b). Otherwise, use that of Figure $9(\mathrm{c})$. Figure 10 gives the $G_{F}$ obtained for the case $F=\left(x_{1}+\bar{x}_{2}+\bar{x}_{4}\right)\left(x_{2}+x_{3}+\bar{x}_{4}\right)\left(\bar{x}_{1}+\bar{x}_{3}\right)\left(x_{2}+x_{3}\right)$.

Theorem 3: Let $F$ be an instance of 3SAT and let $G_{F}$ be the instance of the unit weight multistage graph DVSP obtained using the above construction. For $\delta \geq 4, F$ is satisfiable iff there is a vertex set $X$ such that $d\left(G_{F} / X\right) \leq \delta$ and $|X|=n+2 m-q$, where $m$ is the number of clauses in $F$ and $q$ is the number of two literal clauses. 


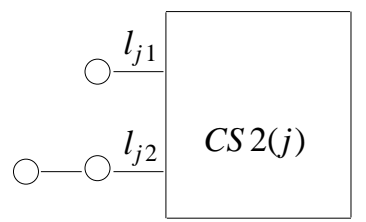

(a) $\left|C_{j}\right|=2$

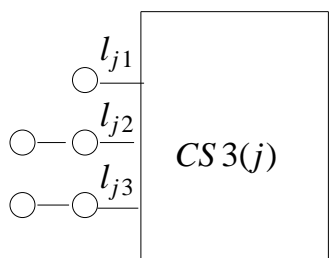

(b) Two unnegated literal

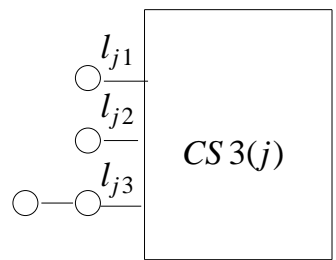

(c) One unnegated literal

Figure 9: Modified clause subassemblies.

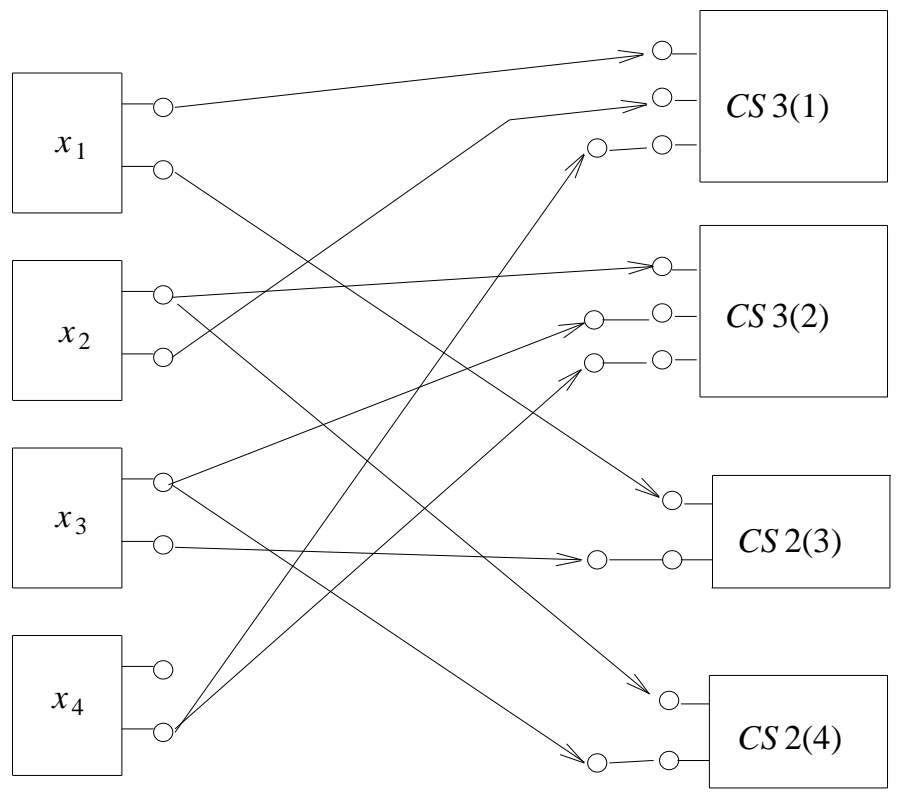

Figure 10: $G_{F}$ for $F=\left(x_{1}+\bar{x}_{2}+\bar{x}_{4}\right)\left(x_{2}+x_{3}+\bar{x}_{4}\right)\left(\bar{x}_{1}+\bar{x}_{3}\right)\left(x_{2}+x_{3}\right)$. 
Proof: Analogous to that of Theorem 1.

\section{Tree DVSP}

In this section we develop a linear time algorithm for the DVSP when the wdag $G$ is a rooted tree. The algorithm is a simple postorder [HORO90] traversal of the tree. During this traversal we compute, for each node $x$, the maximum delay, $D(x)$, from $x$ to any other node in its subtree. If $x$ has a parent $z$ and $D(x)+w(z, x)$ exceeds $\delta$, then the node $x$ is split and $D(z)$ is set to 0 .

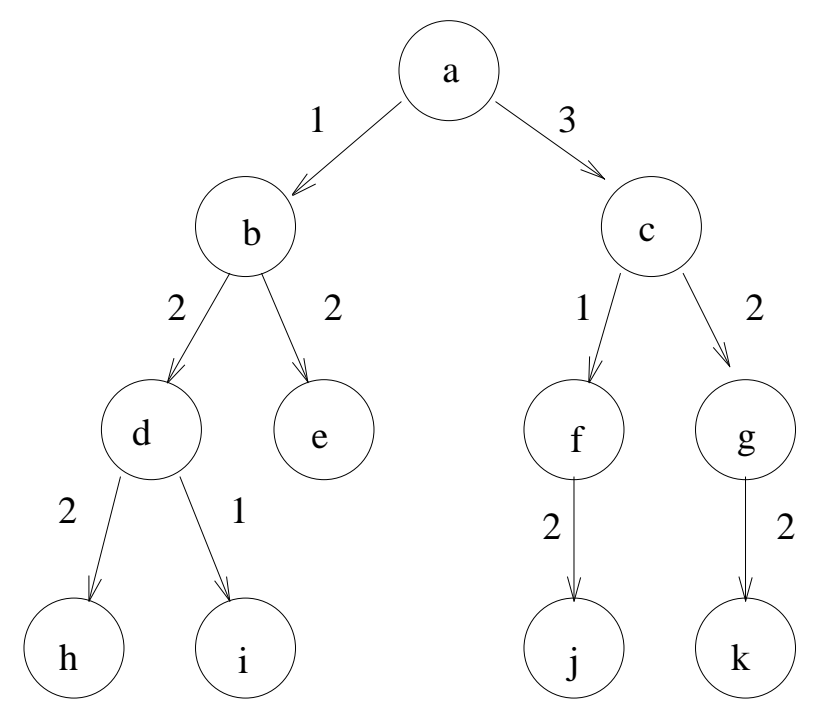

Figure 11: An example tree.

Consider the example tree of Figure 11 and assume $\delta=3$. The delay, $D(x)$, for $x$ a leaf node is 0 . So, $D(x)=0$ for $x \in\{h, i, e, j, k\}$. In postorder, a node is visited after its children have been. When a node $x$ is visited, its delay may be computed as:

$$
D(x)=\max _{y \text { is a child of } x}\{D(y)+w(x, y)\}
$$

So, $D(d)=2$. Since $D(d)+w(b, d)>\delta=3$, we split node $d$ to get the tree of Figure 12(a). Next, $D(b)=2$ and $D(f)=2$ are computed and since $D(b)+w(a, b) \leq 3$ and $D(f)+w(c, f) \leq 3$, neither $b$ nor $f$ is split. Then since $D(g)=2$ and $D(g)+w(c, g)>\delta=3$, node $g$ is split and we get the tree of Figure 12(b). Next, node $c$ is visited and split since $D(c)+w(a, c)=5>3=\delta$. No more nodes are split. The final tree after splitting the three nodes $d, g$, and $c$ is given in Figure 13. The formal algorithm is given in Figure 14. The algorithm assumes that $X$ has been initialized to $\varnothing$ and that 


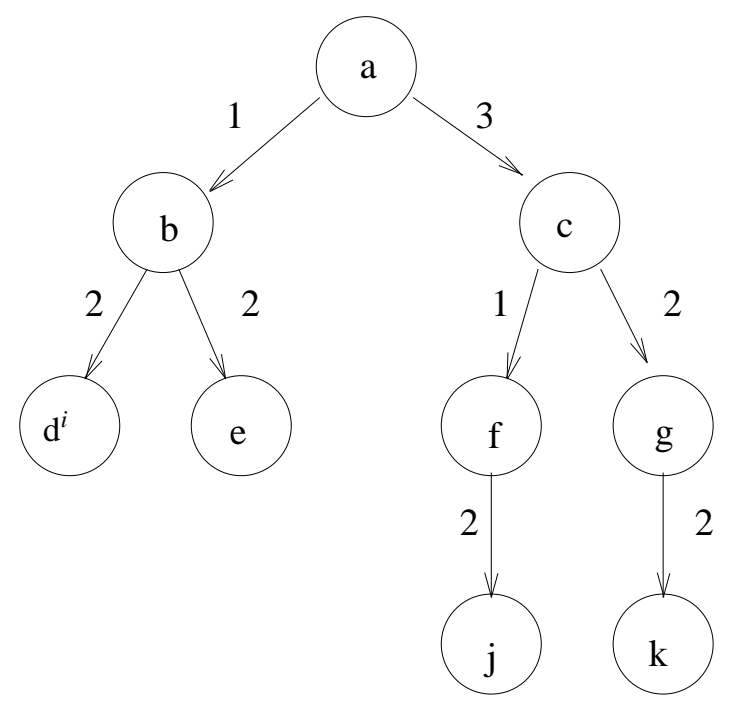

(a)

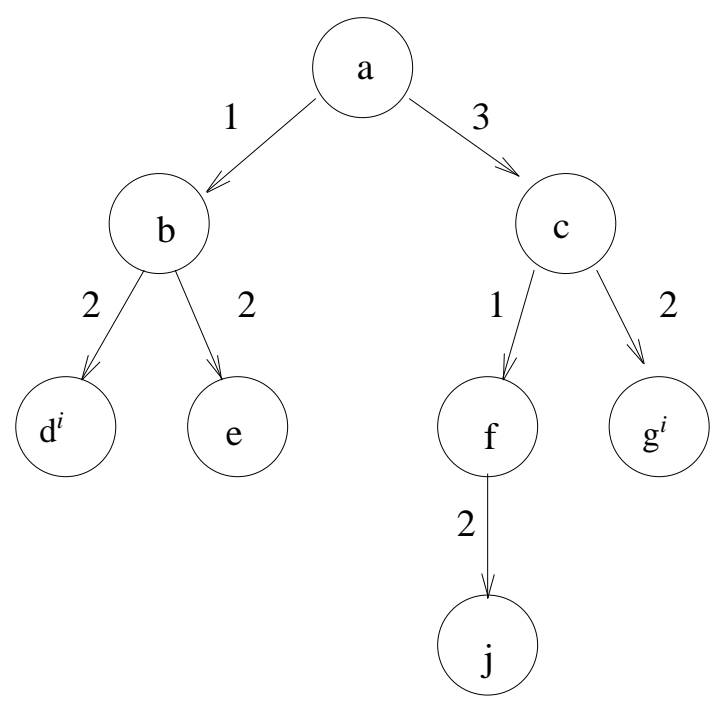

Figure 12: Splitting nodes in Figure 11

$w(i, j) \leq \delta$ for every edge in $T$ since otherwise, there is no solution. Its complexity is $O(n)$ where $n$ is the number of vertices in $T$.

Theorem 4: Procedure DVSP_tree finds a minimum cardinality $X$ such that $d(T / X) \leq \delta$.

Proof: The proof is by induction on the number, $n$, of nodes in the tree $T$. If $n=1$, the theorem is trivially valid. Assume this is so for $n \leq m$ where $m$ is an arbitrary natural number. Let $T$ be a tree with $n+1$ nodes. Let $X$ be the set of vertices split by DVSP_tree and let $W$ be a minimum cardinality vertex set such that $d(T / W) \leq \delta$. We need to show that $|X|=|W|$. If $|X|=0$, this is trivially true. If $|X|>0$, then let $z$ be the first vertex added to $X$ by DVSP_tree. Let $T_{z}$ be the subtree of $T$ rooted at $z$. As $z$ is added to $X$ by DVSP_tree, $D(z)+w($ parent $(z), z)>\delta$. Hence, $W$ must contain at least one vertex $u$ that is in $T_{z}$. If $W$ contains more than one such $u$, then $W$ cannot be of minimum cardinality as $Z=W-\{$ all such $u\}+\{z\}$ is such that $d(T / Z) \leq \delta$. Hence, $W$ contains exactly one such $u$. Let $W^{\prime}=W-\{u\}$. Let $T^{\prime}$ be the tree that results from the removal of $T_{z}$ from $T$ except $z$. If there is a $W^{*}$ such that $\left|W^{*}\right|<\left|W^{\prime}\right|$ and $d\left(T^{\prime} / W^{*}\right) \leq \delta$, then since $d\left(T /\left(W^{*}+\{z\}\right)\right) \leq$ $\delta, W$ is not a minimum cardinality deletion set for $T$. So, $W^{\prime}$ is a minimum cardinality vertex set 


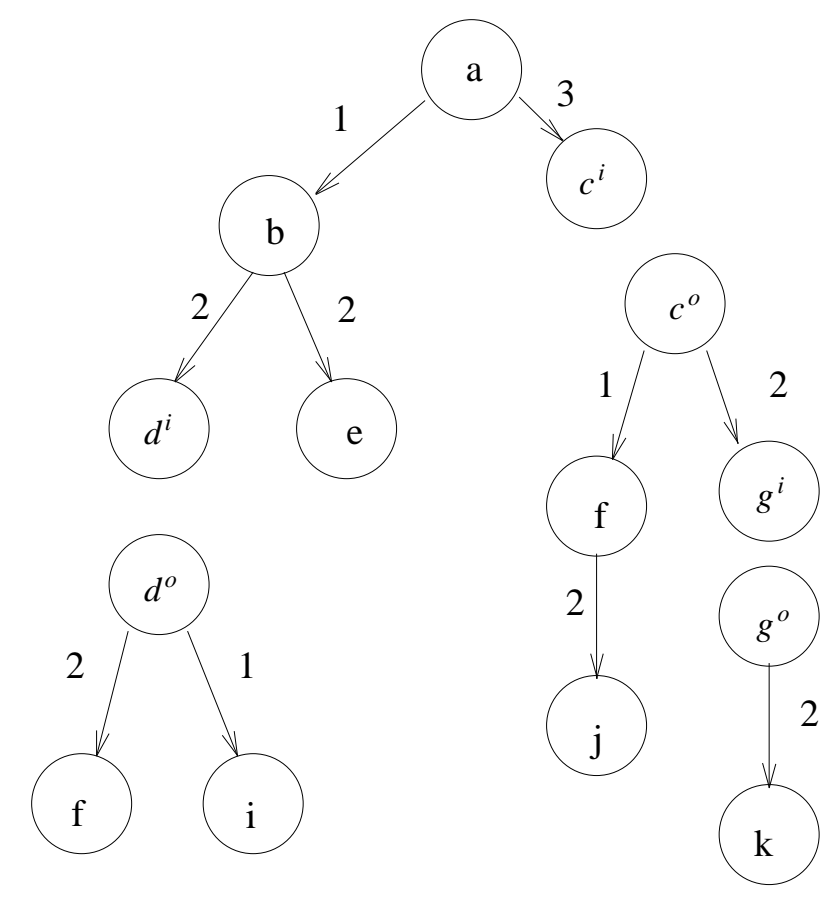

Figure 13: DVSP solution for a tree in Figure 11.

such that $d\left(T^{\prime} / W^{\prime}\right) \leq \delta$. Also, $X^{\prime}=X-\{z\}$ is such that $d\left(T^{\prime} / X^{\prime}\right) \leq \delta$ and furthermore $X^{\prime}$ is the answer produced by DVSP-tree when started with $T^{\prime}$. Since the number of vertices in $T^{\prime}$ is less than $m+1,\left|X^{\prime}\right|=\left|W^{\prime}\right|$. Hence, $|X|=\left|X^{\prime}\right|+1=\left|W^{\prime}\right|+1=|W|$.

\section{A Backtracking Algorithm For DVSP}

Backtracking algorithms [HORO78] generally search a tree organization of the solution space using bounding functions. The solution to our problem is a $0 / 1$ vector $X=\left(x_{1}, x_{2}, \cdots, x_{n}\right)$ where $n$ is the number of vertices and $x_{i}=0$ iff vertex $i$ is not split. We use the binary tree organization used in [HORO78] for the 0/1-knapsack problem. In this organization, the nodes at level $i$ denote a decision on $x_{i}, 1 \leq i \leq n$. If $x_{i}=0$ we move to the left subtree. Otherwise we move to the right subtree of a level $i$ node. Figure 15 shows the solution space tree for the case $n=3$. Each root to leaf path defines a vector $X$ in the solution space.

The remaining features of our backtracking algorithm are :

1) The vertices of the dag are considered in topological order. Thus, $x_{i}$ (of Figure 15) denotes a decision on whether or not the $i$ 'th vertex, in the topological order, of the dag is split. 
procedure DVSP_tree $(T)$;

$\{$ Find minimum cardinality $X$ such that $d(T / X) \leq \delta$ \}

\{Assume that $w(i, j) \leq \delta$ for every edge in $T$ and that

$\{X$ is initialized to $\varnothing\}$

begin

if $T<>$ nil

then begin

$D(T)=0$;

for each child $Y$ of $T$ do

begin

DVSP_tree $(Y)$;

$D(T):=\max \{D(T), D(Y)+w(T, Y)\}$;

end;

if $T$ is not the root

then if $D(T)+w($ parent $(T), T)>\delta$

then begin

$X:=X \cup\{T\} ;\{$ split $T\}$

$D(T)=0$;

end;

end;

end; $\{$ of DVSP_tree $\}$

Figure 14: DVSP algorithm for trees.

2) If the $i$ 'th vertex, in the topological order, is a source or sink vertex then the subtree with $x_{i}$ $=1$ is not considered (i.e., it is eliminated from the tree of Figure 15 ) as source and sink vertices are not to be split.

3) Let $Y$ be a node in the solution space tree. If $Y$ is at level $i$ (root is at level 1 ), then the path from the root to $Y$ determines values for $x_{1}, x_{2}, \cdots, x_{i-1}$. Let $G / / Y$ be the dag obtained from the original dag by splitting the vertices with $x_{j}=1,1 \leq j<i$. Let $f$ be the delay of the maximum delay path in $G / / Y$ that ends at the $i$ 'th vertex in the topological order and let $g(G / / Y)$ be the delay of the maximum delay path in $G / / Y$ that begins at the $i^{\prime}$ th vertex. We use the following rules to move to a child of node $Y$ :

3a) If $f+w(i, j)>\delta$ for some $\langle i, j\rangle \in E$, then set $x_{i}=1$ and eliminate the $x_{i}=0$ subtree.

3b) If $f+g(G / / Y) \leq \delta$, then set $x_{i}=0$ and eliminate the $x_{i}=1$ subtree.

3c) If there is only one edge $\left\langle i, j>\right.$ that leaves vertex $i$, and $f+w(i, j) \leq \delta$, then set $x_{i}=0$ 


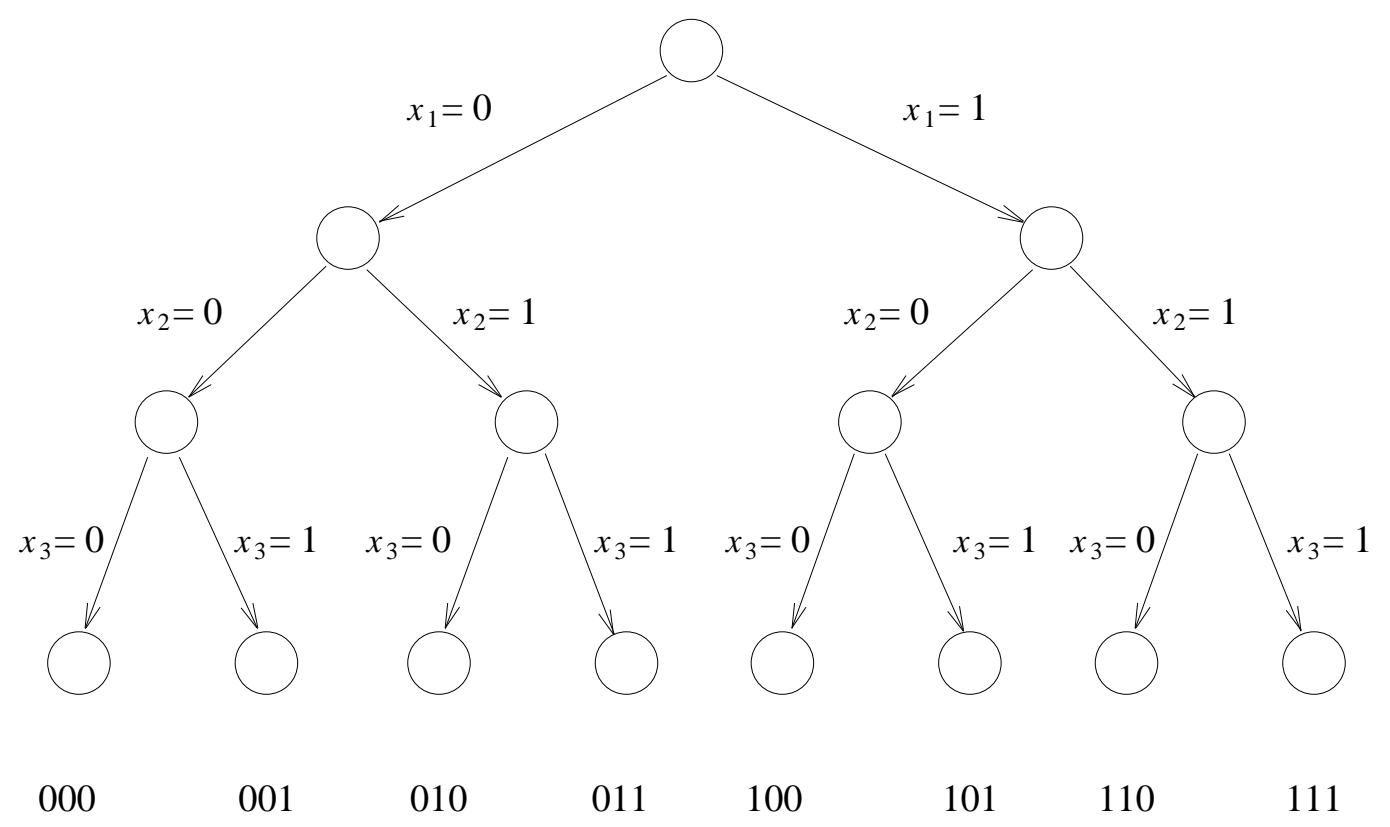

Figure 15: Solution space organization for $n=3$.

and eliminate the subtree $x_{i}=1$.

3d) If none of 3a) - 3d) apply, then search the subtree of $Y$ with $x_{i}=0$ first and later search the one with $x_{i}=1$.

4) To bound a node $Y$, we do the following. Let opt be the number of nodes split in the best solution found so far, and let $r$ be the number of nodes split on the path from the root to $Y$. And let $l(G / / Y)$ be the delay of the maximum delay path in $G / / Y$. It is clear that at least $\Gamma$ $l(G / / Y) / \delta\rceil-1$ additional vertices need to be split. So, if opt $\leq r+\lceil l(G / / Y) / \delta\rceil-1$ then node $Y$ is bounded and the subtree with root $Y$ is not to be searched.

\section{Heuristics For DVSP}

We formulate four simple and intuitively appealing constructive heuristics to obtain a set $X$ such that $d(G / X) \leq \delta$. All four split one vertex at a time until the remaining dag has delay $\leq \delta$. They assume that the input dag has a feasible solution. I.e., no edge has delay $>\delta$.

The first three heuristics have the form given in Figure 16 and differ only in the criteria used to select the next vertex to split. 


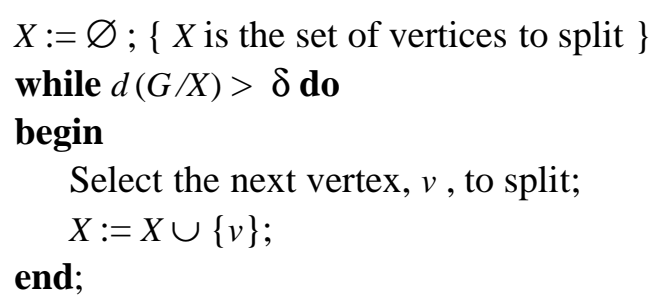

Figure 16: General form of heuristics 1 through 3.

\subsection{Heuristic 1 (h1)}

The selection criteria for the next vertex to split is :

a) $\quad v \notin X$ and $v$ is neither a source nor a sink vertex

b) $\quad v$ is on a path with delay $>\delta$

c) Of all vertices that satisfy a) and b), $v$ has the maximum number of incident edges that are on paths of delay $>\delta$. In case of a tie, let $Z$ be the set of vertices that are tied. For each $u \in$ $Z$ determine $l(u)$ and $r(u)$ such that $l(u)$ is the length of a longest path from a source of $G / X$ to $u$ and $r(u)$ is the length of a longest path from $u$ to a sink of $G / X$. The vertex with the maximum value of $\min \{l(u), r(u)\}$ is selected. If there is still a tie, this is broken arbitrarily.

This heuristic is easily implemented to have run time $O(k(n+e))$ where $k$ is the number of vertices split, $n$ is the number of vertices in the dag, and $e$ is the number of edges in the dag.

\subsection{Heuristic 2 (h2)}

In this heuristic, the next vertex, $v$, to split satisfies criteria a) and b) of Heuristic 1. In addition, the following criteria is employed:

c') Of all the vertices that satisfy a) and b), $v$ is a vertex whose splitting results in a dag that has the fewest number of vertices that are on paths of delay $>\delta$. Ties are broken as in $\mathrm{h} 1$. Heuristic 2 may be implemented to have complexity $O(k n e)$. 


\subsection{Heuristic $3(\mathrm{~h} 3)$}

Heuristic 3 also uses criteria a) and b) used by Heuristic 1. However, criteria c) is replaced by:

c') Of all the vertices that satisfy a) and b), $v$ is such that its splitting results in a dag with least delay. I.e., $v$ is such that $d(G /(X \cup\{v\}))$ is minimum over all choices for $v$. Ties are broken as in h1.

The complexity of Heuristic 3 is $O(k n e)$.

\subsection{Heuristic $4(\mathrm{~h} 4)$}

In this heuristic, the vertices of the dag are examined in two different orders: topological and reverse topological. When the $i$ 'th vertex in the topological (reverse topological) order is examined, it is split if the current dag contains a path comprised solely of vertices $1, \ldots, i$ and one additional vertex that has delay $>\delta$. The heuristic is specified in Figure 17. It can be implemented to run in $O(n+e)$ time. Note that the additional vertex $j$ can be restricted to the set of vertices adjacent to $i$.

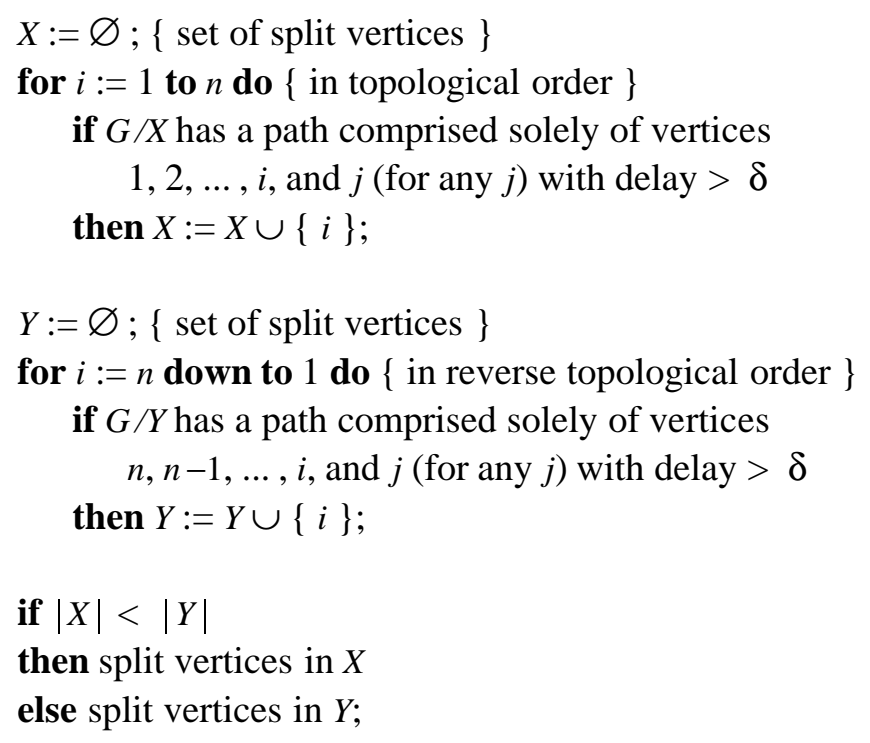

Figure 17: Heuristic 4. 


\section{Experimental Results}

The backtracking algorithm of Section 5 and the four heuristics of Section 6 were programmed in Pascal and run on an Apollo DN3500 workstation. We experimented with two sets of acyclic directed graphs. The first set was obtained from the S-graphs of the ISCAS-89 benchmark sequential circuits [BRGL89]. The S-graphs were first rendered cycle free by the procedure given in [LEE90]. The characteristics of the resulting dags are given in Table 1. The other set of graphs was derived from the ISCAS-85 benchmark combinational circuits [BRGL85]. Here the nodes in the digraph model the gates in the circuit and the edges correspond to the connections between gates. Associated with each edge is the propagation delay along the corresponding circuit gate input. The edge delay was set to the maximum of the rising and falling delays provided in [BRGL85]. The characteristics of these circuits are given in Table 2. For each dag, G, we experimented with the $\delta$ values $\{.9 d(G), .8 d(G), .7 d(G), .6 d(G), .5 d(G), .4 d(G)\}$. Table 3 gives the results for the case $G=\mathrm{s} 400$. Note from Table 1 that $d(\mathrm{~s} 400)=16$. For $\delta$ close to $d(\mathrm{~s} 400)$ (specifically, $\delta=12$ and 14), all four heuristics found optimal solutions. Heuristic 2 was the only one that obtained optimal solutions for all tested $\delta$ values. Table 4 gives the performance of circuit s38584. The backtracking algorithm was able to complete only for the case $\delta=.9 d(G)$ and $\delta$ $=.8 d(G)$ in the time alloted for each run. Heuristic h2 consistently obtained better solutions than obtained by the remaining heuristics. However, its run time, while quite acceptable, was greater than that of heuristics 1 and 4 .

Table 5 gives the results for the combinational circuit $\mathrm{c} 432$. For this circuit, heuristics 2 and 3 found the optimal solution for all tested $\delta$ values. The results for circuit c6288 are given in Table 6. The backtracking algorithm successfully found the optimal solution only for the cases $\delta$ $=287.89=0.9 d(G)$ and $\delta=255.90=0.8 d(G)$. Of the four heuristics, h2 obtained the best solutions for five of the six $\delta$ values tested and h4 was best for the remaining $\delta$ value.

Tables 7 and 8 give the total number of nodes split by each of the four heuristics for each of the sequential and combinational circuits, respectively. For each circuit the six $\delta$ values \{ $.9 d(G), .8 d(G), .7 d(G), .6 d(G), .5 d(G), .4 d(G)\}$ were used and the tables give the sum of the number of vertices split for each of these $\delta$ values. Table 9 and 10 give the $\%$ of tests on which each heuristic obtained the best solution. Heuristic 2, on average, was significantly better than the others.

Tables $11-14$ give the number of nodes split at the two extremes $\delta=0.9 d(G)$ and $\delta=$ $0.4 d(G)$ of the range of $\delta$ values tested. Generally, for $\delta$ close to $d(G)$ the four heuristics tended to obtain solutions of comparable quality while for smaller $\delta$ the differences were more noticeable. However, in all $\delta$ ranges tested, heuristic 2 tended to produce the best solutions. The average run time for each of the circuits and each $\delta$ value is given in Tables 15 and 16. As can be seen heuristics 1 and 4 are very fast. While heuristic 2 is significantly faster than heuristic 3 , it is 
much slower than h1 and h4. Despite this, we recommend h2 because it produces relatively better solutions and its run time is acceptable.

\section{References}

[BRGL85] F. Brglez and H. Fujiwara, "A Neutral Netlist of Ten Combinational Benchmark Circuits and a Target Translator in FORTRAN,"

Proc. IEEE Symp. on Circuits \& Systems, June 1985 pp. 663-666.

[BRGL89] F. Brglez, D. Bryan, and K. Kozminski, "Combinational Profiles of Sequential Benchmark Circuits,"

Proc. of Intern. Symp. on Circuit \& Systems, May 1989, pp. 1929-1934.

[CHEN90] K.T. Cheng and V. D. Agrawal, "A Partial Scan Method for Sequential Circuits with Feedback," IEEE Transactions on Computers, Vol. 39, No. 4, pp. 544-548, April 1990.

[GARE79] M. R. Garey, and D. S. Johnson, "Computers and Intractability", W. H. Freeman and Company, San Francisco, 1979.

[GUPT90] R. Gupta, R. Gupta and M. A. Breuer, "BALLAST: A Methodology for Partial Scan Design," IEEE Transactions on Computers, Vol. 39, No. 4, pp. 538-544, April 1990.

[HORO78] E. Horowitz, and S. Sahni, "Fundamentals of Computer Algorithms", Computer Science Press, Maryland, 1978.

[HORO90] E. Horowitz, and S. Sahni, "Fundamentals of Data Structures in Pascal", Computer Science Press, Maryland, 1990.

[LEE90] D. H. Lee and S. M. Reddy, "On Determining Scan Flip-flops in Partial-scan Designs," Proc. of International Conference on Computer Aided Design, November 1990.

[PAIK90] D. Paik, S. Reddy, and S. Sahni, "Deleting Verticies To Bound Path Lengths", University of Florida, Technical Report, 1990. 


\begin{tabular}{|l|c|c|c|}
\hline circuit & \# vertices & \# edges & $d(G)$ \\
\hline s400 & 173 & 282 & 16 \\
s420 & 37 & 130 & 17 \\
s526 & 27 & 98 & 12 \\
s526n & 27 & 98 & 12 \\
s838 & 69 & 266 & 33 \\
s1423 & 917 & 26 \\
s5378 & 74 & 1314 & 20 \\
s9234 & 233 & 1633 & 32 \\
s13207 & 216 & 3083 & 35 \\
s15850 & 762 & 8562 & 61 \\
s35932 & 608 & 3380 & 36 \\
s38417 & 1777 & 8754 & 29 \\
s38584 & 1396 & 9471 & 129 \\
\hline
\end{tabular}

Table 1: Circuit characteristics (unit delay) of modified sequential circuits

\begin{tabular}{|l|c|c|c|}
\hline circuit & \# vertices & \# edges & max delay \\
\hline c432 & 250 & 426 & 57.40 \\
c499 & 555 & 928 & 53.30 \\
c880 & 443 & 729 & 53.00 \\
c1355 & 587 & 1064 & 49.90 \\
c1908 & 913 & 1498 & 76.59 \\
c2670 & 1426 & 2076 & 86.87 \\
c3540 & 1719 & 2939 & 98.69 \\
c5315 & 2485 & 4386 & 99.30 \\
c6288 & 2448 & 4800 & 319.88 \\
c7552 & 3719 & 6144 & 85.30 \\
\hline
\end{tabular}

Table 2: Circuit characteristics (with max of falling and rising delay) of ISCAS combinational circuits.

\begin{tabular}{|r|r|r|r|r|r|r|r|r|r|r|}
\hline & \multicolumn{7}{|c|}{ \# nodes split } & \multicolumn{5}{c|}{ run time (sec) } \\
\hline$\lfloor\delta\rfloor$ & h1 & h2 & h3 & h4 & optimal & h1 & h2 & h3 & h4 & optimal \\
\hline 14 & 1 & 1 & 1 & 1 & 1 & $<1$ & $<1$ & $<1$ & $<1$ & $<1$ \\
12 & 1 & 1 & 1 & 1 & 1 & $<1$ & $<1$ & $<1$ & $<1$ & $<1$ \\
11 & 2 & 2 & 2 & 3 & 2 & $<1$ & $<1$ & $<1$ & $<1$ & $<1$ \\
9 & 5 & 4 & 4 & 7 & 4 & $<1$ & $<1$ & 1 & $<1$ & 10 \\
8 & 7 & 4 & 4 & 11 & 4 & $<1$ & $<1$ & 1 & $<1$ & 24 \\
6 & 12 & 8 & 11 & 10 & 8 & $<1$ & 1 & 3 & $<1$ & 35980 \\
\hline
\end{tabular}

Table 3: Results for $\mathrm{s} 400$ 


\begin{tabular}{|r|r|r|r|r|r|r|r|r|r|c|}
\hline & \multicolumn{5}{|c|}{ \# nodes split } & \multicolumn{5}{c|}{ run time (sec) } \\
\hline$\lfloor\delta\rfloor$ & h1 & h2 & h3 & h4 & optimal & h1 & h2 & h3 & h4 & optimal \\
\hline 116 & 21 & 2 & 2 & 5 & 2 & 3 & 61 & 61 & $<1$ & 222 \\
103 & 15 & 2 & 4 & 24 & 2 & 2 & 64 & 127 & $<1$ & 17280 \\
90 & 18 & 2 & 5 & 20 & - & 3 & 68 & 181 & $<1$ & - \\
77 & 27 & 4 & 6 & 20 & - & 5 & 127 & 218 & $<1$ & - \\
64 & 40 & 8 & 13 & 27 & - & 7 & 293 & 682 & $<1$ & - \\
51 & 89 & 10 & 37 & 44 & - & 18 & 439 & 2126 & $<1$ & - \\
\hline
\end{tabular}

Table 4: Results for s38584

\begin{tabular}{|c|c|c|c|c|c|c|c|c|c|c|}
\hline & \multicolumn{7}{|c|}{ \# nodes split } & \multicolumn{5}{c|}{ run time (sec) } \\
\hline$\delta$ & h1 & h2 & h3 & h4 & optimal & h1 & h2 & h3 & h4 & optimal \\
\hline 51.66 & 1 & 1 & 1 & 1 & 1 & $<1$ & 1 & 1 & $<1$ & $<1$ \\
45.92 & 2 & 1 & 1 & 9 & 1 & $<1$ & 1 & 1 & $<1$ & $<1$ \\
40.18 & 2 & 2 & 2 & 10 & 2 & $<1$ & 2 & 2 & $<1$ & $<1$ \\
34.44 & 3 & 2 & 2 & 19 & 2 & $<1$ & 2 & 2 & $<1$ & $<1$ \\
28.70 & 4 & 3 & 3 & 10 & 3 & $<1$ & 3 & 3 & $<1$ & 1486 \\
22.96 & 5 & 3 & 3 & 21 & 3 & $<1$ & 4 & 4 & $<1$ & 1007 \\
\hline
\end{tabular}

Table 5: Results for c432

\begin{tabular}{|c|c|c|c|c|c|c|c|c|c|c|}
\hline & \multicolumn{5}{|c|}{ \# nodes split } & \multicolumn{5}{|c|}{ run time $(\mathrm{sec})$} \\
\hline$\delta$ & $\mathrm{h} 1$ & h2 & h3 & h4 & optimal & h1 & $\mathrm{h} 2$ & h3 & $\mathrm{h} 4$ & optimal \\
\hline 287.89 & 69 & 1 & 1 & 2 & 1 & 10 & 158 & 161 & $<1$ & 33 \\
\hline 255.90 & 210 & 3 & 6 & 4 & 2 & 34 & 370 & 764 & $<1$ & 36 \\
\hline 223.91 & 241 & 23 & 33 & 29 & - & 40 & 2634 & 4910 & $<1$ & - \\
\hline 191.92 & 216 & 30 & 86 & 44 & - & 37 & 3603 & 16223 & $<1$ & - \\
\hline 159.94 & 349 & 41 & 86 & 50 & - & 58 & 4886 & 18450 & $<1$ & - \\
\hline 127.95 & 328 & 56 & 86 & 45 & - & 56 & 6971 & 20900 & 1 & - \\
\hline
\end{tabular}

Table 6: Results for c6288 


\begin{tabular}{|l|r|r|r|r|}
\hline circuit & h1 & h2 & h3 & h4 \\
\hline s400 & 28 & 20 & 23 & 33 \\
s420 & 31 & 31 & 31 & 31 \\
s526 & 21 & 33 & 25 & 26 \\
s526n & 21 & 33 & 25 & 26 \\
s838 & 60 & 36 & 36 & 36 \\
s1423 & 58 & 63 & 57 & 62 \\
s5378 & 24 & 9 & 10 & 18 \\
s9234 & 64 & 27 & 33 & 50 \\
s13207 & 139 & 48 & 99 & 87 \\
s15850 & 194 & 59 & 121 & 217 \\
s35932 & 174 & 128 & 147 & 147 \\
s38417 & 414 & 133 & 246 & 406 \\
s38584 & 210 & 28 & 67 & 140 \\
\hline
\end{tabular}

Table 7: Total number of nodes split for modified sequential circuits

\begin{tabular}{|l|r|r|r|r|}
\hline circuit & $\mathrm{h} 1$ & $\mathrm{~h} 2$ & $\mathrm{~h} 3$ & $\mathrm{~h} 4$ \\
\hline $\mathrm{c} 432$ & 17 & 12 & 12 & 70 \\
$\mathrm{c} 499$ & 48 & 72 & 108 & 120 \\
$\mathrm{c} 880$ & 65 & 45 & 97 & 82 \\
$\mathrm{c} 1355$ & 60 & 73 & 97 & 152 \\
c1908 & 128 & 50 & 109 & 144 \\
c2670 & 183 & 59 & 101 & 147 \\
c3540 & 389 & 142 & 262 & 226 \\
c5315 & 260 & 84 & 264 & 184 \\
c6288 & 1413 & 154 & 298 & 174 \\
c7552 & 564 & 249 & 709 & 635 \\
\hline
\end{tabular}

Table 8: Total number of nodes split for combinational circuits 


\begin{tabular}{|l|r|r|r|r|}
\hline circuit & h1 & h2 & h3 & h4 \\
\hline s400 & 50 & 100 & 83 & 33 \\
s420 & 83 & 83 & 83 & 83 \\
s526 & 100 & 33 & 50 & 50 \\
s526n & 100 & 33 & 50 & 50 \\
s838 & 33 & 83 & 83 & 83 \\
s1423 & 83 & 17 & 100 & 33 \\
s5378 & 17 & 100 & 83 & 67 \\
s9234 & 0 & 100 & 50 & 0 \\
s13207 & 0 & 100 & 17 & 17 \\
s15850 & 0 & 100 & 67 & 17 \\
s35932 & 33 & 83 & 0 & 67 \\
s38417 & 17 & 100 & 17 & 17 \\
s38584 & 0 & 79.5 & 55.2 & 39.8 \\
\hline average & 39.7 & & \\
\hline
\end{tabular}

Table 9: Percentage of best solutions for sequential circuits

\begin{tabular}{|c|r|r|r|r|r|r|r|r|r|r|}
\hline & \multicolumn{7}{|c|}{ \# nodes split } & \multicolumn{5}{|c|}{ run time (sec) } \\
\hline circuit & h1 & h2 & h3 & h4 & optimal & h1 & h2 & h3 & h4 & optimal \\
\hline s400 & 1 & 1 & 1 & 1 & 1 & $<1$ & $<1$ & $<1$ & $<1$ & $<1$ \\
s420 & 2 & 2 & 2 & 2 & 2 & $<1$ & $<1$ & $<1$ & $<1$ & $<1$ \\
s526 & 2 & 2 & 2 & 2 & 2 & $<1$ & $<1$ & $<1$ & $<1$ & $<1$ \\
s526n & 2 & 2 & 2 & 2 & 2 & $<1$ & $<1$ & $<1$ & $<1$ & $<1$ \\
s838 & 4 & 4 & 4 & 4 & 4 & $<1$ & $<1$ & $<1$ & $<1$ & $<1$ \\
s1423 & 3 & 3 & 3 & 3 & 3 & $<1$ & $<1$ & $<1$ & $<1$ & 1 \\
s5378 & 1 & 1 & 1 & 1 & 1 & $<1$ & $<1$ & $<1$ & $<1$ & $<1$ \\
s9234 & 4 & 1 & 1 & 2 & 1 & $<1$ & $<1$ & $<1$ & $<1$ & $<1$ \\
s13207 & 5 & 3 & 3 & 3 & 3 & $<1$ & 9 & 10 & $<1$ & 16 \\
s15850 & 5 & 2 & 2 & 2 & 2 & $<1$ & 25 & 25 & $<1$ & 8 \\
s35932 & 10 & 10 & 10 & 10 & - & $<1$ & 127 & 157 & $<1$ & - \\
s38417 & 5 & 3 & 3 & 2 & 2 & $<1$ & 47 & 56 & $<1$ & 10 \\
s38584 & 21 & 2 & 2 & 5 & 2 & 3 & 61 & 61 & $<1$ & 222 \\
\hline
\end{tabular}

Table 10: Sequential circuits, $\delta=0.9 d(G)$ 


\begin{tabular}{|c|r|r|r|r|r|r|r|r|r|r|}
\hline & \multicolumn{7}{|c|}{ \# nodes split } & \multicolumn{5}{c|}{ run time (sec) } \\
\hline circuit & h1 & h2 & h3 & h4 & optimal & h1 & h2 & h3 & h4 & optimal \\
\hline c432 & 1 & 1 & 1 & 1 & 1 & $<1$ & 1 & 1 & $<1$ & $<1$ \\
c499 & 2 & 2 & 6 & 8 & 2 & $<1$ & 9 & 35 & $<1$ & $<1$ \\
c880 & 1 & 1 & 1 & 4 & 1 & $<1$ & 1 & 1 & $<1$ & $<1$ \\
c1355 & 2 & 2 & 5 & 24 & 2 & $<1$ & 11 & 36 & $<1$ & 11 \\
c1908 & 2 & 1 & 1 & 12 & 1 & $<1$ & 7 & 7 & $<1$ & 1 \\
c2670 & 9 & 1 & 1 & 2 & 1 & $<1$ & 10 & 10 & $<1$ & $<1$ \\
c3540 & 17 & 5 & 6 & 10 & - & 1 & 98 & 176 & $<1$ & - \\
c5315 & 13 & 2 & 11 & 4 & 2 & 1 & 52 & 201 & $<1$ & 5 \\
c6288 & 69 & 1 & 1 & 2 & 1 & 10 & 158 & 161 & $<1$ & 33 \\
c7552 & 20 & 5 & 8 & 5 & - & 3 & 163 & 280 & $<1$ & - \\
\hline
\end{tabular}

Table 11: Combinational circuits, $\delta=0.9 d(G)$

\begin{tabular}{|c|r|r|r|r|r|r|r|r|r|r|}
\hline & \multicolumn{7}{|c|}{ \# nodes split } & \multicolumn{5}{|c|}{ run time (sec) } \\
\hline circuit & \multicolumn{1}{|c|}{ h1 } & h2 & h3 & h4 & optimal & h1 & h2 & h3 & h4 & optimal \\
\hline s400 & 12 & 8 & 11 & 10 & 8 & $<1$ & 1 & 3 & $<1$ & 35980 \\
s420 & 7 & 7 & 7 & 7 & 7 & $<1$ & $<1$ & $<1$ & $<1$ & $<1$ \\
s526 & 6 & 13 & 6 & 6 & 6 & $<1$ & $<1$ & $<1$ & $<1$ & $<1$ \\
s526n & 6 & 13 & 6 & 6 & 6 & $<1$ & $<1$ & $<1$ & $<1$ & $<1$ \\
s838 & 8 & 8 & 8 & 8 & 8 & $<1$ & $<1$ & $<1$ & $<1$ & 42 \\
s1423 & 17 & 18 & 16 & 17 & - & $<1$ & 5 & 4 & $<1$ & - \\
s5378 & 6 & 3 & 4 & 3 & 3 & $<1$ & 2 & 2 & $<1$ & $<1$ \\
s9234 & 17 & 13 & 16 & 25 & - & $<1$ & 13 & 17 & $<1$ & - \\
s13207 & 40 & 16 & 35 & 24 & - & 2 & 85 & 247 & $<1$ & - \\
s15850 & 68 & 23 & 49 & 78 & - & 11 & 513 & 1059 & $<1$ & - \\
s35932 & 64 & 37 & 55 & 38 & - & 6 & 1064 & 2018 & $<1$ & - \\
s38417 & 160 & 60 & 115 & 190 & - & 25 & 1924 & 8553 & $<1$ & - \\
s38584 & 89 & 10 & 37 & 44 & - & 18 & 439 & 2126 & $<1$ & - \\
\hline
\end{tabular}

Table 12: Sequential circuits, $\delta=0.4 d(G)$ 


\begin{tabular}{|r|r|r|r|r|r|r|r|r|r|c|}
\hline & \multicolumn{5}{|c|}{ \# nodes split } & \multicolumn{5}{|c|}{ run time (sec) } \\
\hline circuit & \multicolumn{1}{|r|}{ h1 } & h2 & h3 & h4 & optimal & h1 & \multicolumn{1}{c|}{ h2 } & h3 & h4 & optimal \\
\hline c432 & 5 & 3 & 3 & 21 & 3 & $<1$ & 4 & 4 & $<1$ & 1007 \\
c499 & 18 & 21 & 30 & 40 & - & $<1$ & 99 & 187 & $<1$ & - \\
c880 & 33 & 20 & 39 & 25 & - & $<1$ & 41 & 112 & $<1$ & - \\
c1355 & 18 & 21 & 25 & 56 & - & $<1$ & 117 & 170 & $<1$ & - \\
c1908 & 40 & 21 & 66 & 41 & - & 1 & 214 & 724 & $<1$ & - \\
c2670 & 57 & 34 & 56 & 64 & - & 3 & 662 & 1339 & $<1$ & - \\
c3540 & 113 & 48 & 86 & 68 & - & 10 & 1867 & 5100 & $<1$ & - \\
c5315 & 95 & 40 & 105 & 91 & - & 12 & 2408 & 8612 & $<1$ & - \\
c6288 & 328 & 56 & 86 & 45 & - & 56 & 6971 & 20900 & 1 & - \\
c7552 & 189 & 129 & 372 & 330 & - & 40 & 23933 & 83417 & $<1$ & - \\
\hline
\end{tabular}

Table 13: Combinational circuits, $\delta=0.4 d(G)$

\begin{tabular}{|l|r|r|r|r|}
\hline circuit & $\mathrm{h} 1$ & $\mathrm{~h} 2$ & $\mathrm{~h} 3$ & $\mathrm{~h} 4$ \\
\hline $\mathrm{c} 432$ & 33 & 100 & 100 & 17 \\
$\mathrm{c} 499$ & 100 & 33 & 0 & 17 \\
$\mathrm{c} 880$ & 33 & 83 & 50 & 0 \\
$\mathrm{c} 1355$ & 100 & 17 & 0 & 33 \\
c1908 & 0 & 100 & 17 & 0 \\
c2670 & 0 & 100 & 50 & 33 \\
c3540 & 0 & 100 & 0 & 0 \\
c5315 & 0 & 100 & 0 & 17 \\
c6288 & 0 & 100 & 0 & 17 \\
c7552 & 0 & 81.6 & 23.4 & 17 \\
\hline average & 26.6 & & & 15.1 \\
\hline
\end{tabular}

Table 14: Percentage of best solutions. 


\begin{tabular}{|l|r|r|r|r|}
\hline circuit & $\mathrm{h} 1$ & $\mathrm{~h} 2$ & $\mathrm{~h} 3$ & $\mathrm{~h} 4$ \\
\hline $\mathrm{s} 400$ & $<1$ & $<1$ & 1 & $<1$ \\
$\mathrm{~s} 420$ & $<1$ & $<1$ & $<1$ & $<1$ \\
$\mathrm{~s} 526$ & $<1$ & $<1$ & $<1$ & $<1$ \\
$\mathrm{~s} 526 \mathrm{n}$ & $<1$ & $<1$ & $<1$ & $<1$ \\
$\mathrm{~s} 838$ & $<1$ & $<1$ & $<1$ & $<1$ \\
$\mathrm{~s} 1423$ & $<1$ & 3 & 3 & $<1$ \\
$\mathrm{~s} 5378$ & $<1$ & 1 & 1 & $<1$ \\
$\mathrm{~s} 9234$ & $<1$ & 3 & 5 & $<1$ \\
$\mathrm{~s} 13207$ & 1 & 37 & 95 & $<1$ \\
$\mathrm{~s} 15850$ & 5 & 195 & 379 & $<1$ \\
$\mathrm{~s} 35932$ & 2 & 532 & 749 & $<1$ \\
$\mathrm{~s} 38417$ & 11 & 681 & 2671 & \\
$\mathrm{~s} 38584$ & 6 & 175 & 558 & \\
\hline
\end{tabular}

Table 15: Average run time for sequential circuits.

\begin{tabular}{|l|r|r|r|r|}
\hline circuit & $\mathrm{h} 1$ & $\mathrm{~h} 2$ & $\mathrm{~h} 3$ & $\mathrm{~h} 4$ \\
\hline $\mathrm{c} 432$ & $<1$ & 2 & 2 & $<1$ \\
$\mathrm{c} 499$ & $<1$ & 59 & 112 & $<1$ \\
$\mathrm{c} 880$ & $<1$ & 14 & 36 & $<1$ \\
$\mathrm{c} 1355$ & $<1$ & 70 & 101 & $<1$ \\
$\mathrm{c} 1908$ & 1 & 84 & 194 & $<1$ \\
$\mathrm{c} 2670$ & 1 & 168 & 320 & $<1$ \\
$\mathrm{c} 3540$ & 6 & 875 & 2186 & $<1$ \\
$\mathrm{c} 5315$ & 5 & 682 & 2607 & $<1$ \\
$\mathrm{c} 6288$ & 39 & 3104 & 10235 & $<1$ \\
$\mathrm{c} 7552$ & 18 & 6212 & 22632 & \\
\hline
\end{tabular}

Table 16: Average run time for combinational circuits. 